\title{
Lógicas de intervención estatal en vacíos urbanos ferroviarios: los casos de Pro.Cre.Ar Ciudad y Distrito Sustentable, Mendoza Argentina
}

\author{
Julián Quiroga Ríos ${ }^{1}$ \\ Universidad Nacional de Cuyo \\ Consejo Nacional de Investigaciones Científicas y Técnicas
}

@ [j.quirogarios@conicet.gov.ar ]

\section{Rodrigo Martín Magallanes²}

Universidad Nacional de Cuyo Consejo Nacional de Investigaciones Científicas y Técnicas

@ [ tinchomaga@gmail.com ]

Cita sugerida: Quiroga Ríos, J. y Magallanes, R. M. (2021). Lógicas de intervención estatal en vacíos urbanos ferroviarios: los casos de Pro.Cre.Ar Ciudad y Distrito Sustentable, Mendoza Argentina. Revista Huellas, Volumen 25, № 2 , Instituto de Geografía, EdUNLPam: Santa Rosa. Recuperado a partir de: http:// cerac.unlpam.edu.ar/index.php/huellas

DOI: http://dx.doi.org/10.19137/huellas-2021-2517

\begin{abstract}
Resumen
A lo largo de la historia urbana las ciudades han sido fundamentales para garantizar la reproducción de las formas de acumulación vigentes. En la actualidad, Estado y mercado desempeñan papeles complementarios para la maximización de rentas urbanas a favor de este último. En este sentido, el presente trabajo pretende dar cuenta de dos formas de intervención urbanas fuertemente fomentadas por el Estado local pero que se diferencian en aspectos sustanciales promoviendo articulaciones políticas particulares que impactan en el territorio generando rupturas urbanísticas, político-institucionales, simbólicas y ambientales. De esta forma, a partir del análisis del programa habitacional estatal Pro. Cre.Ar y del proyecto Distrito Sustentable, daremos cuenta de las prácticas del Estado municipal que inciden en la estructura urbana de la Ciudad de Mendoza, Argentina. Consideramos aquí que estas prácticas se encuentran en línea con las políticas urbanas de la ciudad que generan condiciones favorables para la captación de rentas urbanas por parte de agentes privados.
\end{abstract}

Palabras clave: Área Metropolitana de Mendoza; Estado local; Planificación urbana 


\title{
Logics of State intervention in urban railway vacant lots: the cases of Pro.Cre.Ar Ciudad \& Distrito Sustentable, Mendoza Argentina
}

\begin{abstract}
Throughout urban history, cities have been instrumental in ensuring the reproduction of existing forms of accumulation. At present, State and market play complementary roles in the maximization of urban income in favour of the latter. In this respect, this work aims to account for two forms of urban intervention strongly fostered by the local State, which differ from one another in substantial aspects, promoting particular political articulations that impact on the territory generating urban, political, institutional, symbolic and environmental ruptures. In this way, based on the analysis of the State habitational program Pro.Cre.Ar and the Distrito Sustentable project, we will account for the local State practices that affect the urban structure of the Ciudad de Mendoza, Argentina. We hold that these practices are related to the city's urban politics that generate favorable conditions for the raising of urban income by private agents.
\end{abstract}

Keywords: Metropolitan Area of Mendoza; Local State; Urban planning

\section{Lógicas de intervenção estadual em lugares urbanos ferroviários vazios: Os casos Pro.Cre.Ar Ciudad e Distrito Sustentable, Mendoza, Argentina}

\section{Resumo}

Ao longo da história urbana as cidades foram fundamentais para garantir a reprodução das formas de acumulação existentes. Na atualidade, Estado e mercado têm papeis complementários para maximizar as rendas urbanas em favor deste último. Neste sentido, este trabalho pretende advertir sobre as duas formas de intervenção urbana favorecidas pelo Estado local, mas que se diferenciam em aspectos substanciais promovendo articulações políticas particulares que impactam no território gerando rupturas urbanísticas, políticoinstitucionais, simbólicas e ambientais. Desta forma, a partir da análise do programa Pro.Cre.Ar e do projeto Distrito Sustentable, informaremos sobre as práticas do Estado municipal que incidem na estrutura urbana da cidade de Mendoza, Argentina. Consideramos que estas práticas se encontram alinhadas com as políticas urbanas da cidade as quais geram condições favoráveis para a capitalização das rendas urbanas por parte dos agentes privados.

Palavras-chave: Área metropolitana de Mendoza; Estado local; Planificação urbana 


\section{Introducción}

Desde mediados de los setenta la orientación neoliberal en las políticas económicas y sociales ha marcado profundamente la forma en la que las ciudades han sido y son producidas, en estrecha relación con los distintos matices que adoptan los modos de acumulación en la región.

Así, las ciudades han resultado fundamentales para garantizar la reproducción de las formas de acumulación capitalistas. Este proceso se ha llevado a cabo con distintos matices en diferentes países. Principalmente en los denominados dependientes y aún entre ellos, estas políticas de reestructuración no se han aplicado de la misma forma, verificando trayectorias diferenciales que obligan a pensar los procesos neoliberales insertados contextualmente (Theodore, Peck, and Brenner, 2009).

En este sentido, en el presente trabajo nos proponemos analizar dos grandes intervenciones urbanas en el último vacío urbano de la Ciudad de Mendoza, Argentina ${ }^{3}$; el programa habitacional Pro.Cre.Ar Ciudad y el gran proyecto urbano (GPU) Distrito Sustentable. Ambos se encuentran emplazados en espacios que quedaron vacantes luego de la privatización y posterior cierre de los ferrocarriles para transporte de pasajeros en Argentina.

A lo largo de los años la zona ha sido objeto de diferentes intentos de apropiación. Tempranamente y en consonancia con las consecuencias sociales de los programas de ajuste estructural, se instalaron de manera informal asentamientos que vieron en este vacío urbano el lugar para habitar. Dada la ubicación privilegiada y su alto valor inmobiliario, se proyectó un emprendimiento ferro-urbanístico con las características de Puerto Madero en Buenos Aires ${ }^{4}$, fuertemente impulsado por el Estado en sus diferentes escalas.

Por medio de un exhaustivo análisis documental (normativas urbanas, Plan de Ordenamiento Territorial Provincial y Municipal, Plan de Renovación y Reactivación Urbana municipal) y lectura de bibliografía especializada, intentaremos dar cuenta de las formas en las que el Estado, principalmente local, interviene en la estructura urbana de la ciudad mediante la activación de proyectos urbanísticos de alto impacto.

Para alcanzar los objetivos planteados, tomamos y adaptamos como herramienta metodológica la propuesta que hace Vainer (2012) para analizar a los Grandes Proyectos Urbanos (GPU) desde un enfoque crítico. El autor sostiene que en los GPU se pueden reconocer una serie de intervenciones urbanas que instauran rupturas en la ciudad. Según el carácter de las mismas, dichas rupturas pueden ser analizadas desde la dimensión ${ }^{5}$ política/institucional, que hace referencia al reconocimiento de grupos dominantes, como también de las nuevas alianzas, institucionalidades y 
formas de gobierno urbano emergentes. También se pueden analizar desde las normativas inmobiliarias, expresadas en la habilitación de nuevos usos del suelo y las modificaciones de indicadores urbanísticos como el Factor de Ocupación total (FOT) y el Factor de Ocupación del Suelo (FOS) que introduce el GPU. A su vez, se reconoce la dimensión urbanístical paisajística, que tiene ver con el impacto sobre la estructura urbana local; la económica/financiera, relacionada con la apropiación y distribución del costo beneficio, la estructuración de proyectos y las modalidades de financiamiento; la simbólica, relacionada con las nuevas representaciones de ciudad que genera y formas de consumo del espacio emergentes. Y, por último, la dimensión socio-ambiental, que hace referencia a la existencia de espacios verdes, impactos ambientales y sociales, y la existencia de desplazamiento de población.

Así, intentaremos reconocer las diferentes articulaciones políticas que dieron lugar a arreglos urbanísticos particulares (Del Río, 2017), que son entendidos como la conjunción de las capacidades político-institucionales y factores tanto limitantes como habilitantes, que posibilitaron cada uno de los casos de estudio.

En primera instancia avanzaremos en una caracterización de las tendencias urbanas del Área Metropolitana de Mendoza (AMM) en general y de la Ciudad de Mendoza en particular. En segundo lugar, analizaremos la historia de los terrenos del ex Ferrocarril (FFCC) General San Martín, fundamental para comprender las intervenciones urbanas analizadas. En tercer lugar, nos abocaremos al caso de estudio Pro.Cre.Ar Ciudad, indagando sobre las características del programa a escala nacional y los impactos en el área de estudio. En cuarto lugar, nos centraremos en el análisis del caso Distrito Sustentable como ejemplo de la política urbana desarrollada por el municipio. Por último, en las conclusiones, se retoman las ideas principales abordadas a lo largo del trabajo con el foco puesto en las similitudes y diferencias de cada caso en las cuales se evidencia el tipo de ciudad que cada escala estatal pondera.

\section{Características urbanas del AMM y la Ciudad de Mendoza}

El Área Metropolitana de Mendoza (AMM) se encuentra localizada en el Oasis Norte de la Provincia homónima, se posiciona como el principal aglomerado del oeste argentino y el cuarto más grande del país. Está conformada por los espacios urbanos de los departamentos de Ciudad de Mendoza, Guaymallén, Godoy Cruz, Las Heras, Maipú y Luján de Cuyo. Actualmente ocupa una superficie del 0,1\% del territorio de la provincia, concentra el $65 \%$ de la población total (936.224 habitantes para el año 2016) y el $71 \%$ de la población urbana (CIPPEC, 2018, p. 25). 
Figura $N^{0} 1$. Ubicación geográfica de la Ciudad de Mendoza y el área de estudio.

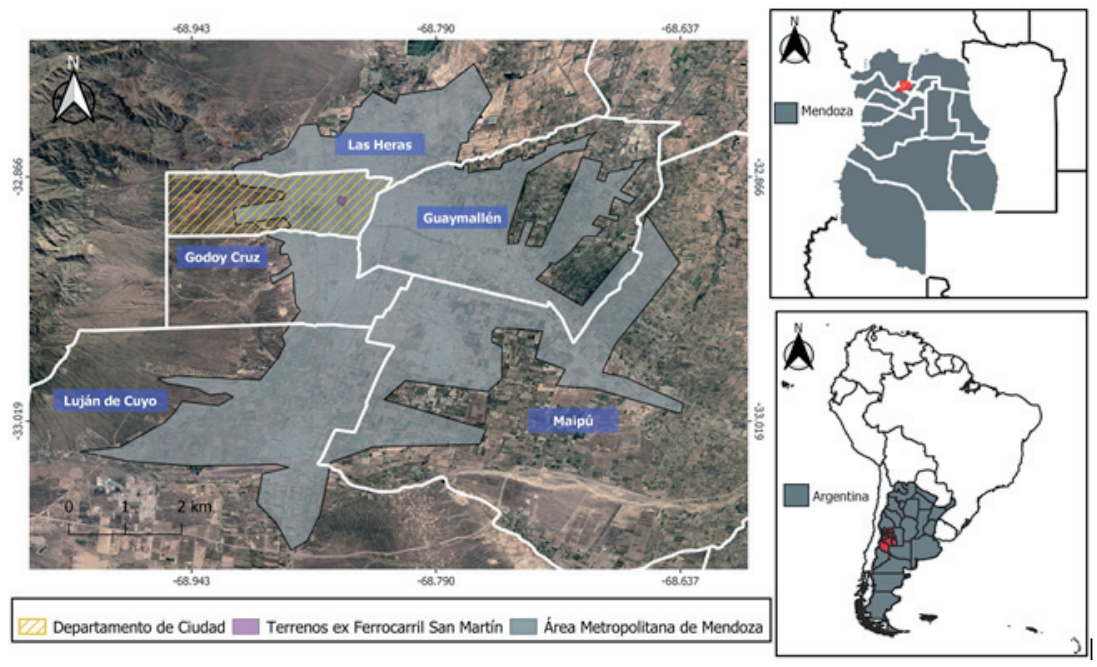

Fuente: Elaboración en conjunto con el Geógrafo Luis Verdugo.

En los últimos cincuenta años el AMM ha experimentado una fuerte expansión de la mancha urbana. La principal característica de este modelo de crecimiento es que se produce de forma desordenada, descontrolada, siguiendo una lógica especulativa y permitiendo que la urbanización avance sobre tierras agrícolas de alto valor ambiental (Gudiño et al., 2014, p.8).

Desde la década del noventa, bajo la lógica del empresarialismo urbano (Harvey 1989), se han producido transformaciones en las ciudades, entre ellas es posible mencionar: un crecimiento ininterrumpido de distintas modalidades de barrios cerrados (Molina, 2013; Roitman, 2000), junto con un fuerte aumento de la ocupación informal en la periferia urbana (Lentini, Palero, and Montaña, 2010); la expulsión de villas o asentamientos populares de la zona céntrica (Insa, 2010; Morgani and Rizzo, 2014).

El Gobierno local ha logrado posicionar a la ciudad como polo turístico nacional e internacional, a través del marketing urbano promocionándola con slogans como "Ciudad Maravillosa" o "Capital Internacional del Vino". Esto permitió el arribo de inversiones directas extranjeras (IDE) especializadas en el rubro turismo (cadenas hoteleras como Park Hyatt, Sheraton, etc), y fue acompañado por un fuerte proceso de embellecimiento urbano y políticas de seguridad y vigilancia (Morgani and Raffani, 2012).

Frente a este modelo de crecimiento desordenado, en el año 2009 se promulgó la Ley Provincial N8051 de Ordenamiento Territorial y de Usos del Suelo con el objetivo de contribuir al ordenamiento del territorio como procedimiento político-administrativo comandado por el Estado. El objeti- 
vo principal de la norma se centra en lograr un desarrollo más equilibrado y sostenible en los espacios urbanos y rurales. A escala metropolitana se plantea la necesidad de tender hacia una ciudad compacta, que detenga el crecimiento difuso hacia la periferia y lograr una mayor densificación de áreas centrales, ocupando baldíos existentes (D'Inca and Berón, 2013).

En el año 2016 se crea un nuevo órgano de gestión interjurisdiccional metropolitano, promovido por la Universidad Nacional de Cuyo, denominado UNICIPIO. El mismo tiene como objetivo la articulación y el trabajo conjunto de todos los municipios que componen el AMM, para abordar de forma integrada las problemáticas socio-ambientales. Su creación permitió que la metrópolis se incorpore a la Iniciativa de Ciudad Emergentes y Sostenibles (ICES) propuesta por el Banco Interamericano de DesarroIlo (BID), conformándose de esta forma la alianza estratégica públicoprivada para el desarrollo y ejecución de obras, respondiendo al modelo importado de ciudad sostenible propuesto por el organismo multilateral (Magallanes, 2017).

En los últimos cinco años se han multiplicado las obras públicas de renovación urbana en la Ciudad de Mendoza, en su gran mayoría financiadas por el BID. A partir de la articulación política entre municipio, provincia y nación, se implementó el Plan de Renovación Urbana (20162021) que permitió contar con los fondos para renovar luminaria, calles, bulevares, plazas, parques degradados, construcción de paseos culturales turísticos y renovación de espacios culturales.

\section{Vacíos urbanos en la ciudad: los ex terrenos del FFCC Gral. San Martín}

Las políticas de ajuste estructural desarrolladas en Argentina, iniciadas a finales de los años setenta y profundizadas en la década de los noventa, produjeron diversos impactos a nivel territorial; la generación de vacíos urbanos en áreas en desuso (Cuenya 2012) fue una de las características que asumió dicho proceso en la Ciudad de Mendoza. Tal es el caso de los terrenos del ex Ferrocarril General San Martín (Figura № 2), que comprende 36 hectáreas ubicadas en el centro la ciudad que quedaron vacantes luego del proceso de privatización. La localización privilegiada y el alto valor inmobiliario de los mencionados terrenos han provocado a lo largo de los últimos 30 años, diferentes acciones y prácticas por parte de diversos agentes sociales que buscan desarrollar sus lógicas de apropiación del espacio urbano. En este sitio se emplazan los dos casos en análisis, el Distrito Sustentable y Pro.Cre.Ar Ciudad.

Durante la etapa neoliberal destructiva (década de los noventa), que implica el desmantelamiento (parcial) de acuerdos políticos e institucionales por el reemplazo de "iniciativas reformadoras orientadas al mercado" 
(Theodore et al. 2009, p.6), los terrenos fueron ocupados por tres asentamientos informales de considerable envergadura (Costa Esperanza, Eschorihuela y Malargüe), y existieron organizaciones culturales (Casa Amérika) y sindicales (Unión Ferroviaria) que lucharon contra la privatización del espacio público. A fines de la década del noventa, los terrenos que pertenecían al Estado Nacional fueron cedidos a la Ciudad de Mendoza para iniciar el proceso de re funcionalización a partir del desarrollo de distintos proyectos urbanos. Comenzó con la construcción del moderno Parque Central (2005), continuó con la venta de terrenos aledaños al parque a desarrolladores inmobiliarios para la construcción de torres de alta gama (Casa Magna) y un hipermercado (Coto). En el año 2012, el Gobierno Provincial inauguró la estación central del nuevo sistema de transporte metropolitano Metrotranvía, y en el 2019 se amplía el ramal hacia Las Heras atravesando toda el área.

Figura № 2. Ubicación de Pro.Cre.Ar Ciudad y Distrito Sustentable, Mendoza, Argentina

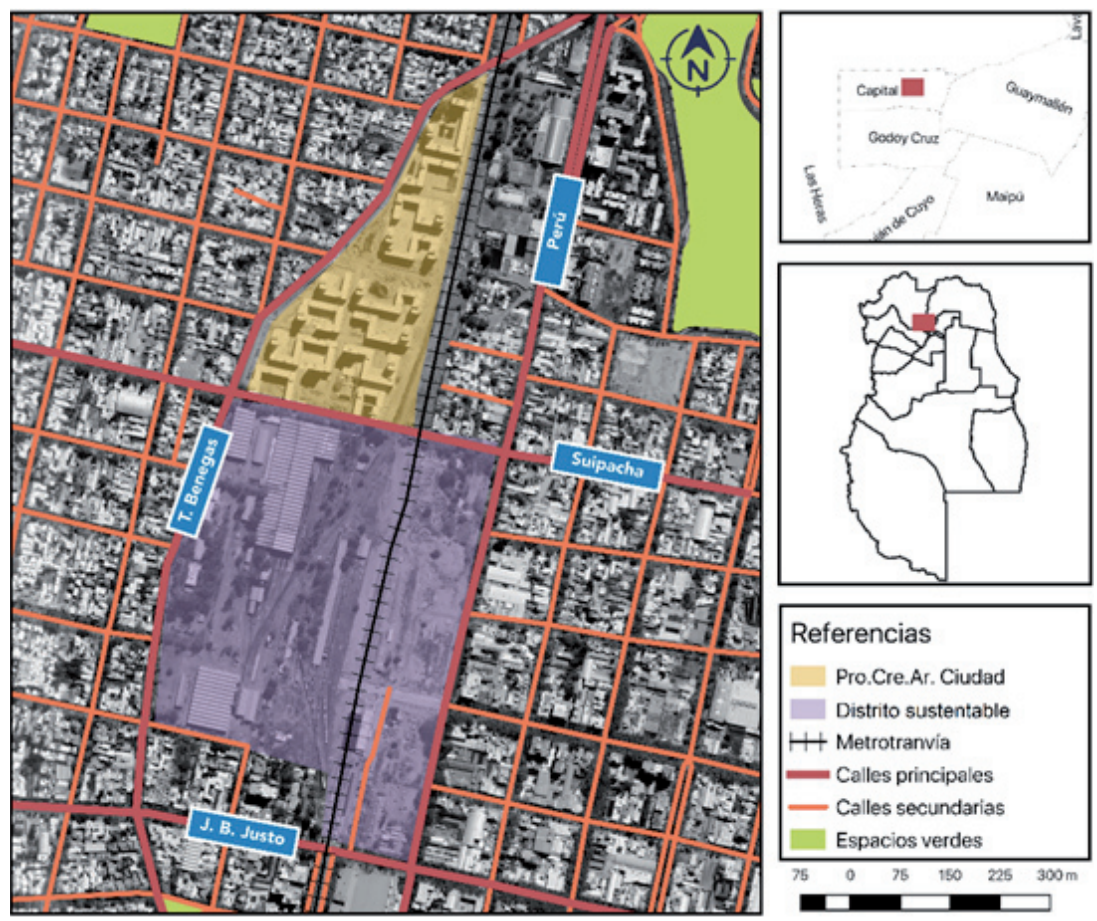

Fuente: Elaboración propia.

En el año 2002 la Corporación Antiguo Puerto Madero Sociedad Anónima (CAPMSA) presentó el proyecto Mendoza-Madero. Esta propuesta 
público-privada desató un conflicto con las organizaciones sociales mencionadas y con los habitantes del asentamiento, generando ciclos especulativos "que desencadenan violentos procesos de exclusiones de mayorías, con elevados costos sociales, ambientales, económicos y políticos para la comunidad" (Salomone and Marsonet, 2011, p.11).

Hacia 2009 la fuerte presión social derivada de la conflictividad llevó a que CAMPSA desista de su proyecto urbanístico. Sin embargo, de este proceso es necesario rescatar el escenario favorable que generó para futuros emprendimientos. Esto principalmente porque en el año 2007 mediante la ordenanza municipal 3.694/17433/07 se establecieron los nuevos criterios urbanísticos para los ex terrenos del FFCC. Entre otros aspectos la ordenanza habilitaba nuevos usos (residencial y residencial mixto; comerciales, culturales y de servicios) y tal vez el cambio más significativo, se habilitaron para la zona densidades muy superiores a las del resto del área cercana (hasta 600 habitantes por hectárea) (Jajamovich, 2016, p.72).

El nuevo cuerpo normativo sirvió para que posteriormente se pudieran realizar otros proyectos en la zona, uno netamente habitacional como el desarrollo urbanístico Pro.Cre.Ar Ciudad, y el proyecto de renovación urbana Distrito Sustentable (ver Figura $N^{\circ} 2$ ). Complementariamente otorgó al Estado municipal las condiciones necesarias para avanzar en la erradicación de los asentamientos informales, principal obstáculo para la concreción de los proyectos en la zona.

\section{El Pro.Cre.Ar Ciudad y las tenciones en la política habitacional}

El programa Pro.Cre.Ar Bicentenario es una política federal del Gobierno Nacional lanzada en 2012 que proyectó la entrega de 400 mil créditos hipotecarios para la construcción, compra de terreno y construcción, ampliación, terminación y refacción de viviendas. También, para adquirir unidades habitacionales producidas por el propio programa en sus desarrollos urbanísticos, como en el caso de estudio que se propone. Si bien el programa apuntaba a solucionar el acceso a la vivienda de sectores relegados por el mercado, fue principalmente una medida anticíclica pensada para impulsar la economía y generar fuentes de empleo (Segura and Cosacov, 2019, p.3).

Con el cambio en el gobierno nacional en 2015, que implicó el fin de más de una década de gobierno del Frente para la Victoria (FPV), el programa se reformuló. Se generan nuevas líneas de crédito, se adoptan nuevos criterios en relación a los procesos de selección y al papel estatal (financiamiento y subsidio), y se introduce un nuevo tipo de crédito hipotecario -los UVA (Unidad de Valor Adquisitivo)- como principal herramienta financiera del programa. 
Si bien no es el objetivo de este trabajo indagar sobre el papel de los créditos UVA, sí es necesario hacer algunas menciones pertinentes. En primer lugar, el alto grado de demanda de este tipo de créditos radica principalmente en las bajas cuotas iniciales sustentadas en un sistema de indexación, tanto de la cuota como del capital, en base a dos indicadores principales: IPC (Índice de Precios al Consumidor) y CER (Coeficiente de Estabilización de referencia) ${ }^{6}$.

Dadas las bajas expectativas en relación a la inflación (IPC) con las que se promocionaba el programa pero que no tuvieron luego correlato con la realidad, los beneficiarios de este tipo de crédito se vieron profundamente perjudicados. Como ilustra Galván (2020, p.46), si bien el índice de morosidad en las cuotas es bajo, dado que se efectúa por débito automático, la solvencia de los beneficiarios se vio menguada en otros aspectos como recortes en gastos de recreación, transporte o con el sobre-endeudamiento familiar.

En segundo lugar, la institucionalización de esta herramienta crediticia en el mayor programa de viviendas del Estado y la retracción de este último, como por ejemplo en el otorgamiento del subsidio ${ }^{7}$, marca el perfil que se impuso en materia de políticas públicas en Argentina posterior al año 2015. Las nuevas regulaciones otorgan a los agentes privados, en especial a las entidades bancarias, un papel protagónico en la estructuración de las políticas públicas urbanas.

En definitiva, si el Pro.Cre.Ar I fue concebido como una política integral que apuntaba a solucionar el acceso a la vivienda de sectores medios y como una herramienta anticíclica (Segura and Cosacov, 2019); la segunda versión del programa se basó en regulaciones estatales que, por una parte, retrajeron el papel estatal y, por otra, otorgaron mayores beneficios al sector financiero no productivo.

El Pro.Cre.Ar Ciudad ${ }^{8}$ (Figura $N^{\circ} 3$ ) no estuvo exento de los cambios institucionales que se adoptaron en el programa posterior al año 2015. Si bien la línea de desarrollos urbanísticos fue la que más tardó en aplicarse, principalmente debido a dificultades referidas al acceso a suelo urbano y a conflictos sociales en las ubicaciones elegidas (Canestraro, 2016; Segura and Cosacov, 2019), el proyecto en Mendoza fue uno de los primeros en avanzar. En gran medida gracias a la disponibilidad de suelo urbano bien ubicado y a la articulación política directa entre el Estado Nacional y municipal.

En efecto, de las 11.000 viviendas que se otorgaron el 2012 en todo el territorio nacional, el departamento de Capital logró captar el 12\% (Diario Unidiversidad, 12 de junio 2017). Las obras del proyecto comenzaron en el año 2014 luego de que se lograra la liberalización del terreno por parte de las familias que allí se habían asentado de manera informal. 
Figura $N^{\circ}$ 3. Fotografía aérea Desarrollo Urbano Pro.Cre.Ar Ciudad

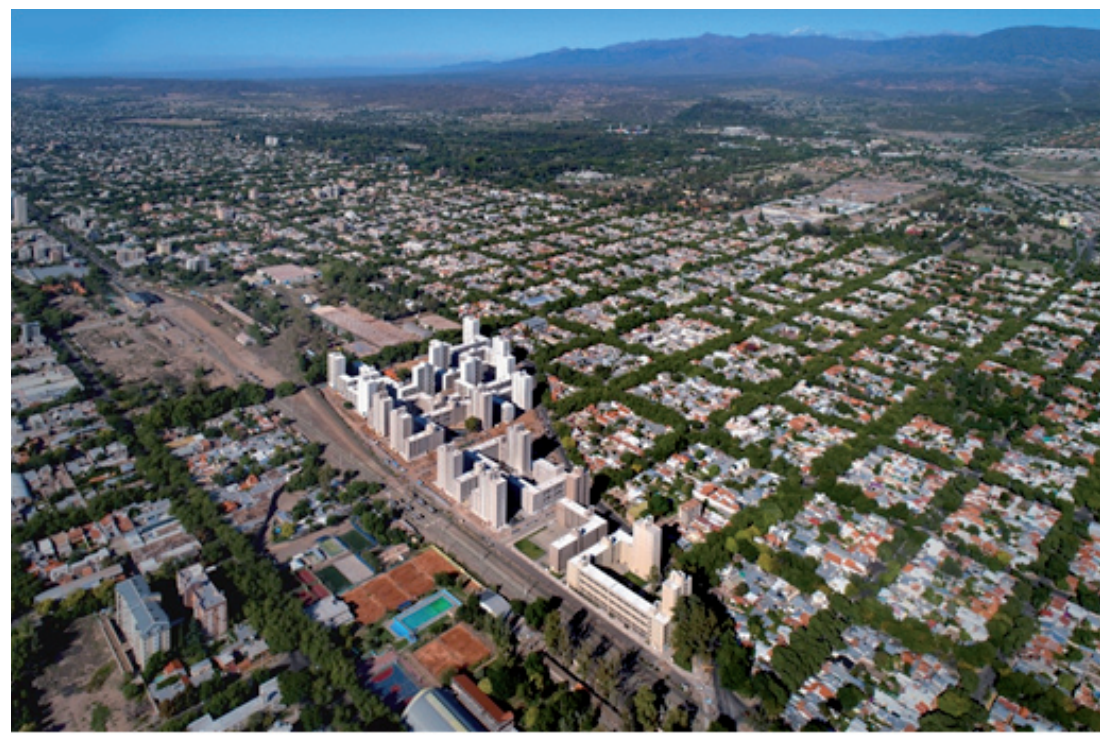

Fuente: Ministerio Desarrollo Territorial y Hábitat, disponible en: https://www.argentina.gob. ar/habitat/procrear/desarrollosurbanisticos/mendoza-mendoza-capital).

La articulación político-institucional entre funcionarios nacionales y municipales fue clave en el proceso de implementación del Pro.CreAr Ciudad que contó, como ya se ha mencionado, con los cambios normativos promovidos con anterioridad en los terrenos del ex FFCC y por otro, con suelo urbano de calidad y bien ubicado cedido por el municipio al Programa ${ }^{9}$. De esta manera, el Estado municipal se convierte en un actor fundamental en la movilización de rentas urbanas (Cuenya et al. 2012) que impacta en la estructura de precios de inmuebles y suelo urbano en la zona circundante.

En definitiva, la cesión de tierras por parte del Estado municipal, las innovaciones institucionales-normativas y los vínculos personales entre funcionarios nacionales y municipales marcaron el arreglo urbanístico particular (Del Río, 2017) que permitió la implementación del proyecto. Luego, en el marco del giro hacia la completa mercantilización del Programa (2015-2019), existieron intenciones de profundizar dicha tendencia fuertemente fomentada por el nuevo gobierno nacional y el Estado municipal, ahora del mismo partido político.

Si bien la construcción del complejo habitacional comenzó en 2014 y terminó a fines de 2017, recién en agosto de 2019 se entregaron las primeras viviendas. Durante ese periodo la institucionalización de los cré- 
ditos UVA, entendidos como una regulación mercantilizada de la política habitacional, se encontraba en pleno auge.

Este periodo, en el cual los departamentos se encontraban terminados pero no adjudicados, sirvió a la retención con fines especulativos que aumentó su precio de mercado. Esta situación fue la que motivó a que funcionarios del gobierno local (municipal y provincial) junto con las empresas constructoras comenzaron un proceso en el cual se buscó instalar la necesidad de vender las unidades por fuera del programa, alegando que los beneficiarios del Pro.Cre.Ar no podrían solventar los nuevos costos de los departamentos.

Esta idea que tendía a profundizar la mercantilización de Pro.Cre.Ar (a escala local), fuertemente impulsada por las empresas constructoras que querían formar parte de la comercialización, no llegó a concretarse. Sin embargo, estas acciones de lobby o cabildeo (Hofman and Aalbers, 2017), sirvieron para dar cuenta del desfasaje entre los montos de los créditos del programa y los nuevos precios de mercado de los inmuebles, que sí se tradujo en una modificación mercantil (a escala nacional), de los criterios del préstamo al permitir subir los montos otorgados por las entidades bancarias privadas ${ }^{10}$.

En definitiva, una vez institucionalizados los créditos UVA al interior del Pro.Cre.Ar y salvados los desfasajes entre precio de la vivienda y crédito, los departamentos de la "mini ciudad de la sexta" fueron adjudicados en un $80 \%$ en menos de un año según datos aportados por Dirección de Infraestructura de la Municipalidad de Capital. Es importante destacar que, si bien el Estado municipal fue fundamental para la concreción de la obra, en la actualidad su rol es simplemente el de mantenimiento de la zona (alumbrado, barrido y limpieza) según datos aportados por la dirección arriba mencionada y la Dirección de Vivienda.

\section{El Gran Proyecto Urbano mediante Articulación Público-Privada: Distrito Sustentable}

En el año 2018 fue relanzado por la Municipalidad de la Ciudad de Mendoza, en el marco del Plan de Renovación Urbana, el GPU que terminará de urbanizar las 24 hectáreas aún vacantes del predio del Ferrocarril General San Martín. Esto sucede nueve años después de que CAMPSA desistió del desarrollo de Mendoza Madero. Durante ese tiempo existieron cambios en las condiciones económicas, financieras, políticas y sociales que permitieron destrabar los conflictos emergentes de aquel entonces.

De manera sintética podemos nombrar algunos hitos fundamentales que acontecieron: a) el surgimiento de la alianza UNICIPIO-BID y su pertenencia a la ICES; b) el lobby gobierno local-gobierno nacional-Agencia 
de Administración de Bienes del Estado (AABE); c) el avance de la densificación con la construcción de las torres PROCREAR que revalorizaron el área; d) las modificaciones del Código urbano de edificación y usos del suelo logradas previamente en el proyecto Mendoza Madero; y e) el debilitamiento y/o desaparición de organizaciones que resistieron en el anterior proceso.

Tal como sostiene Cuenya \& Corral (2011) los GPU tienen la capacidad de generar un aumento extraordinario de la rentabilidad del suelo por el hecho de localizarse en áreas estratégicas que les permite formar parte de un mercado a escala internacional. Hay tres características fundamentales en cuanto a su origen, que coinciden con la propuesta urbanística a desarrollarse en el área: 1) usualmente es el sector público quien lleva a cabo las inversiones en infraestructura para acondicionar el área (servicios públicos y obras viales); 2) generan modificaciones en los usos del suelo y densidades edilicias, permitiendo implantar nuevos usos; 3) promueve grandes inversiones de actores privados que desarrollan emprendimientos inmobiliarios y construyen edificios de alta gama.

Para entender este proceso es importante reconocer que existen espacios de lobby o cabildeos (Hofman and Aalbers, 2017) entre instituciones públicas y privadas en distintas escalas que favorecieron el avance del proyecto. Es por eso que un actor clave es la alianza UNICIPIO-BID, ya que logra posicionar al Área Metropolitana de Mendoza (AMM) en la iniciativa ICES, y así avanzar con el objetivo de implementar el modelo de ciudad sostenible que propone el organismo multilateral, incluidos en la Agenda 2030 de la CEPAL para el Desarrollo Sostenible ${ }^{11}$. Como sostiene Sánchez y Moura (2005), estos modelos urbanos son postulados como buenas prácticas que deben ser imitadas por otras ciudades para transformarse en dinámicas y competitivas, capaz de sostener el desarrollo de una economía global.

Los argumentos están comprendidos en el documento "Plan de Acción Área Metropolitana de Mendoza Sostenible (2018)", donde existe una gran diversidad de agentes públicos y privados participantes, que dan cuenta del cabildeo interescalar. Dicho plan fue elaborado de forma conjunta entre el UNICIPIO, BID, Gobierno de Mendoza y el Gobierno Nacional, a través Ministerio del Interior, Obras Públicas y Vivienda de la Presidencia de la Nación; a su vez, brindaron asesorías consultoras privadas de prestigio internacional en materia de sustentabilidad ${ }^{12}$, participaron universidades (Universidad Nacional de Cuyo y Universidad Tecnológica Nacional) e importantes empresas locales, así como también la Fundación Nuestra Mendoza ${ }^{13}$. En el mencionado documento se presenta la metodología propuesta por el BID para llevar a cabo un diagnóstico del AMM y se elabora el plan de acción con los ejes prioritarios de desarrollo. 
El Distrito Sustentable (figura No 4) se encuentra incluido en el eje "Intervenciones de transformación urbana" presentado con el nombre "Plan de intervención y puesta en valor de los terrenos del ex Ferrocarril General San Martín". Este designa a la Municipalidad de la Ciudad como actor clave para su desarrollo en el corto y mediano plazo, donde se expone la idea de planificación para poner en valor el patrimonio y mejorar la conectividad.

Figura $N^{\circ} 4$. Planos de la propuesta ganadora del Distrito Sustentable

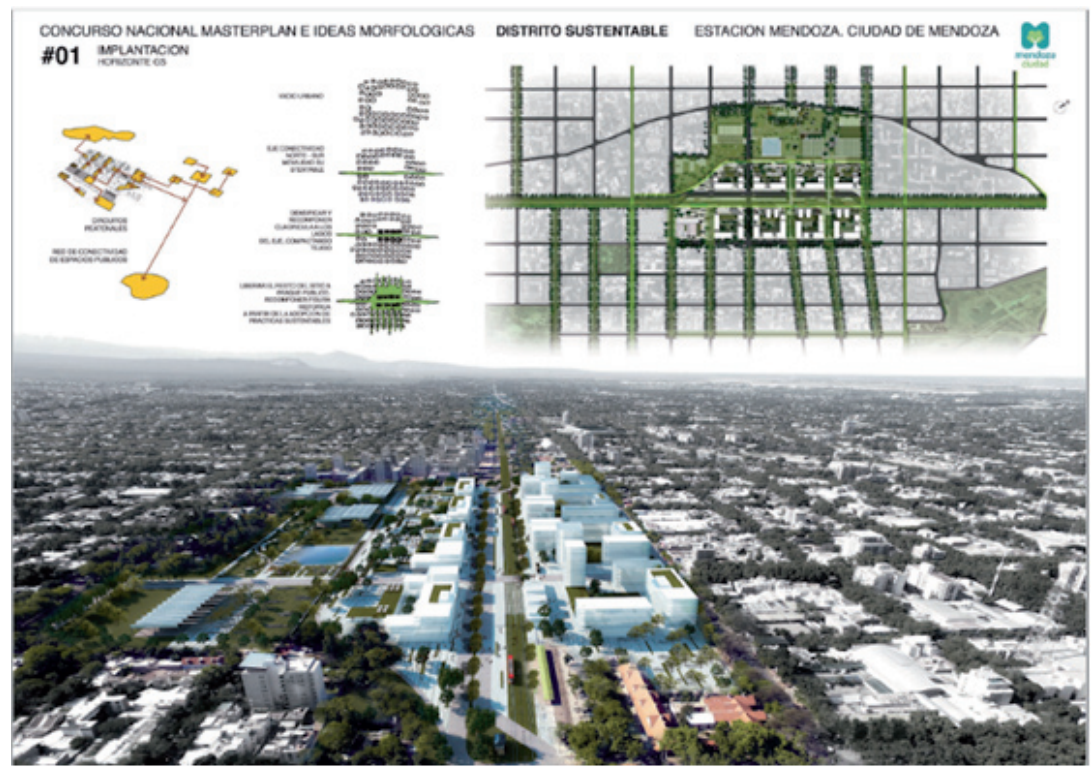

Fuente: Plataforma de Arquitectura, disponible en: https://www. plataformaarquitectura.cl/ cl/871116/estacion-mendoza-la-sustentabilidad-como-guia-para-la-generacion-de-espaciopublico).

La propuesta, novedosa para Mendoza, se basa en la recuperación patrimonial de antiguos galpones ferroviarios y el desarrollo de una ciudad más inclusiva y sustentable. Plantea la reutilización del gran baldío urbano a partir de una mixtura de usos del suelo comercial, cultural-patrimonial, habitacional y espacios verdes; complementariamente, escalona el conjunto edilicio hacia el espacio del parque y la cordillera, que cumple un rol identitario fundamental ${ }^{14}$.

Tal como se puede apreciar en la figura 4, este GPU generará un quiebre desde el punto de vista urbanístico y arquitectónico con respecto al área circundante, reconfigurando la imagen de la Ciudad de Mendoza y estableciendo un nuevo centro urbano (ver figura $\mathrm{N}^{\circ} 4$ ). 
La iniciativa responde a argumentos propios de urbanizaciones sobresalientes que profundizan la elitización del espacio, enriqueciendo la cultura de emprendedores/as y consumidores/as sustentables, principalmente dirigida a sectores de alto poder adquisitivo. Como novedad, el proyecto propone la construcción de mercados verdes, huertas orgánicas, edificios con terrazas verdes y la instalación de un museo del vino.

En cuanto a la dimensión político-institucional, también fueron claves las articulaciones políticas entre Gobierno local, provincial, nacional y la Agencia de Administración de Bienes del Estado (AABE). Durante el gobierno de Mauricio Macri (2015-2019) existieron relaciones fluidas con el Gobierno de Mendoza, presidido por Alfredo Cornejo y la Municipalidad de Mendoza dirigida por Rodolfo Suarez (actual gobernador provincial).

Este lobby político fue promovido en conjunto por el gobierno nacional y municipal, logrando que la AABE permita dar comienzo al proceso de urbanización de los terrenos. Se estableció como acuerdo general que el gobierno nacional se encargaría de subastar los terrenos; la ganancia obtenida se invertirá para dotar de infraestructura al predio y para el traslado de los viejos talleres al distrito de Palmira, en el departamento de San Martín (página web municipalidad de la Ciudad de Mendoza, 22-11-2018).

En este modelo de gestión urbana empresarial, el gobierno local asume el rol de promotor ya que, mediante un modelo de desarrollo sostenible y un llamado explícito a invertir en la ciudad, genera condiciones favorables para que los agentes privados puedan obtener mayor rentabilidad del suelo. El Estado desde su rol como regulador intenta imponer una nueva propuesta urbanística ad hoc, flexible y ajustada a sus propias reglas, en donde se articulan las escalas locales, nacionales e internacionales (Vainer, 2012). Asimismo, al generar obras de mantenimiento e infraestructura, logra acceder a fondos otorgados por el BID para el embellecimiento urbano.

Para acceder a los créditos externos es necesario generar acuerdos institucionales que posibiliten la idea de "ciudades financiables". Como sostiene (Fiori Arantes, 2006) hay cuatro nuevas formas institucionales que exigen los organismos multilaterales: creación de agencias privadas, asociaciones público-privadas (APP), captura de recursos del mercado crediticio nacional e internacional y creación de bonos distritales.

En la presentación del GPU, la Municipalidad de la Ciudad de Mendoza explicita que la única forma de lograr este proyecto es mediante el modelo de asociación público y privado. Hasta el momento sólo existe el Plan Maestro, que es el instrumento que determina la nueva morfología, como también estipulados los costos y beneficios económicos. Tal como plantean autores especialistas en la temática (Cuenya and Corral 2011; Vainer 2012), los Planes Maestros asumen un carácter ideológico donde se expresa una imagen urbana que el gobierno local debe exhibir a los 
distintos agentes intervinientes. A partir de allí se producirán las negociaciones entre sector público y privado, y la creación de entes y autoridades específicas para la reurbanización del área ferroviaria.

\section{Consideraciones finales}

A lo largo del trabajo hemos podido analizar dos formas distintas de intervenciones urbanas por parte del Estado en lo que constituye el último gran vacío urbano de la Ciudad de Mendoza. Ambos casos analizados permiten avanzar en la comprensión de las políticas públicas urbanas, teniendo presente el perfil que adquieren las intervenciones estatales en el territorio que dan lugar a la producción de un tipo particular de ciudad.

En este sentido, ambos casos de estudio generaron arreglos urbanísticos particulares que introdujeron rupturas en las dimensiones analíticas planteadas y en la estructura urbana de la Ciudad de Mendoza. El cuadro № 1 sintetiza los puntos más relevantes de ambos casos.

Cuadro $\mathbf{N}^{\circ} 1$. Síntesis de las principales rupturas a partir de las dimensiones analíticas

\begin{tabular}{|c|c|c|}
\hline $\begin{array}{l}\text { Dimensión } \\
\text { analítica }\end{array}$ & Pro.Cre.AR & Distrito Sustentable \\
\hline $\begin{array}{c}\text { Político/ } \\
\text { institucional }\end{array}$ & $\begin{array}{l}\text { Articulación política directa entre } \\
\text { Estado Nacional y Municipal- } \\
\text { Nueva articulación entre Estado } \\
\text { Nacional y bancos privados-Imple- } \\
\text { mentación e institucionalización de } \\
\text { los créditos UVA }\end{array}$ & $\begin{array}{c}\text { Conformación de la unidad de } \\
\text { gestión metropolitana UNICIPIO- } \\
\text { Alianza entre UNICIPIO y Banco } \\
\text { Interamericano de Desarrollo- Arti- } \\
\text { culación Estado Nacional (AABE) } \\
\text { y Estado Municipal }\end{array}$ \\
\hline $\begin{array}{c}\text { Normativa } \\
\text { inmobiliaria }\end{array}$ & \multicolumn{2}{|c|}{$\begin{array}{l}\text { Densificación del área-Cambio en las alturas máximas permitidas-Habili- } \\
\text { tación de nuevos usos del suelo comercial, espacios verdes, administrati- } \\
\text { vo y habitacional- Revalorización de área degradada y nuevas estructu- } \\
\text { ras de precios del suelo }\end{array}$} \\
\hline $\begin{array}{l}\text { Urbanística/ } \\
\text { paisajística }\end{array}$ & \multicolumn{2}{|c|}{$\begin{array}{l}\text { Impacto en la dinámica de la Ciudad de Mendoza a partir de la genera- } \\
\text { ción de nuevas centralidades -Cambios en la trama urbana: apertura de } \\
\text { nuevas calles, generación de espacio verdes y medios de transporte sus- } \\
\text { tentable (Metrotranvía-red de ciclovías)-Desarrollo en altura-Reutilización } \\
\text { de galpones patrimoniales }\end{array}$} \\
\hline $\begin{array}{c}\text { Económica/ } \\
\text { financiera }\end{array}$ & $\begin{array}{l}\text { Mayor protagonismo de entidades } \\
\text { financieras bancarias a partir del } \\
\text { año 2015- Solvencia de la pobla- } \\
\text { ción perjudicada por la estructura } \\
\text { de los créditos hipotecarios UVA }\end{array}$ & $\begin{array}{l}\text { Gerenciamiento público/privado } \\
\text { del Gran Proyecto Urbano - } \\
\text { Financiamiento externo del Banco } \\
\text { Interamericano de Desarrollo }\end{array}$ \\
\hline
\end{tabular}




\begin{tabular}{|c|c|c|}
\hline \multirow{3}{*}{ Simbólica } & $\begin{array}{c}\text { Recuperación de la población } \\
\text { residente en áreas centrales- } \\
\text { Ampliación de acceso a la ciudad } \\
\text { para sectores sociales medios- Je- } \\
\text { rarquización del espacio }\end{array}$ & $\begin{array}{c}\text { Tendencia hacia un prototipo de } \\
\text { ciudad sostenible- Jerarquización } \\
\text { y elitización del espacio-Nuevas } \\
\text { pautas de consumo }\end{array}$ \\
\cline { 2 - 3 } & $\begin{array}{c}\text { Ponderación positiva del centro de la ciudad para atraer inversiones y } \\
\text { residentes }\end{array}$ \\
\hline \multirow{2}{*}{$\begin{array}{c}\text { Social/ } \\
\text { ambiental }\end{array}$} & $\begin{array}{c}\text { Desplazamiento de asentamientos informales y de organizaciones sindi- } \\
\text { cales y culturales. Renovación y mayor demanda de servicios de agua, } \\
\text { luz y cloacas. Aumento de vigilancia urbana. }\end{array}$ \\
\cline { 2 - 2 } & \multicolumn{2}{|c|}{$\begin{array}{c}\text { Incorporación de productos inmo- } \\
\text { biliarios sustentables y espacio } \\
\text { verdes }\end{array}$} \\
\hline
\end{tabular}

Fuente: elaboración propia.

Las articulaciones políticas en ambos casos difieren en aspectos centrales. En el caso de Pro.Cre.Ar, se destaca la articulación política directa entre el Estado Nacional y Municipal para posibilitar el acceso a la vivienda de sectores de ingresos medios. Mientras que en el caso del Distrito Sustentable, la creación de la alianza inter-municipal (UNICIPIO) fue como condición necesaria para la captación de fondos de organismos internacionales con el fin de generar ciudades sustentables.

Estas modificaciones político-institucionales permitieron avanzar con los dos proyectos cambiando normas urbanísticas o haciendo uso de las disposiciones normativas del proyecto trunco de Mendoza-Madero. Si bien en ambos proyectos el estado municipal es protagonista, el caso del Distrito Sustentable marca una nueva forma de gestión urbana, basada en un fuerte papel del sector privado en el marco de los APP. En el caso de Pro.Cre.Ar Ciudad, aun cuando también coincide con los lineamientos políticos de densificar la ciudad, al ser un programa de escala nacional, el estado municipal asume el papel de gestionar ciertos aspectos logrando capitalizar la imagen positiva que genera la entrega de viviendas.

Los nuevos lineamientos en materia económica y financiera en los casos de estudio son relevantes y sintomáticos del interés de la clase dominante y de su poder de influencia en el Estado. La institucionalización de los créditos hipotecarios UVA, las bajas expectativas inflacionarias con las que se promocionaba este tipo de crédito para captar más "beneficiarios", la solvencia de la población que se ve perjudicada por la estructura del crédito UVA, la creciente importancia de los flujos de capital aportados por organismos internacionales y las adecuaciones político-institucionales 
que se requieren para acceder a dicho mercado de capitales (UNICIPIO por ejemplo), son algunas de las características centrales que demuestran la tendencia hacia una plena mercantilización de la política urbana, característica central de la urbanización capitalista (Pírez and Cosacov, 2016) y que en la actualidad acentúa sus tintes financieros.

Ambas transformaciones generan un quiebre urbanístico-paisajístico presentando una nueva imagen de la Ciudad de Mendoza que impacta en los usos del suelo y las formas de consumo. En los dos casos, con sus particularidades, son una muestra del proceso de vuelta al centro de la Ciudad que implica el retorno de capitales públicos y privados, como así también de discursos a dichas áreas (Navarrete, Avila, and Bernabeu, 2017). Es por eso que el BID con su propuesta ciudad sustentable, al menos desde lo discursivo, se transforma en la propuesta más innovadora y permeable para el modo de gestión empresarial.

Por último, se producen externalidades negativas que suelen traer aparejado los GPU en relación a procesos de exclusión, segregación, desplazamiento de asentamientos informales. Socialmente, se genera una nueva lógica de consumo sustentable, relacionada con la "onda verde", que enmascara el proceso de elitización de áreas centrales renovadas.

En definitiva, si bien los casos de estudio difieren en aspectos sustantivos, ilustran la forma que adquieren las políticas urbanas en un contexto regional de re-estructuración neo-liberal. En éstas se evidencia que las regulaciones, en especial aquellas que dependen del Estado municipal, tienen una marcada lógica mercantil en la cual son los agentes privados quienes marcan la orientación de las políticas urbanas, en una tendencia hacia la plena mercantilización de las mismas. Así, el proyecto de desarrollo urbano propuesto por el Estado municipal, pondera un arreglo urbanístico particular el cual se caracteriza por una articulación política directa entre dicha escala estatal y privados, en especial organismos internacionales de crédito.

\section{Referencias bibliográficas}

Canestraro, María Laura. (2016). Sobre El Derecho a La Ciudad y El Acceso Al Suelo Urbano. Reflexiones a Partir de Intervenciones Estatales Recientes (Mar Del Plata, 2012-2015). ESTUDIOS SOCIOTERRITORIALES. Revista de Geografía (20):57-74.

CIPPEC. 2018. Hacia El Desarrollo Urbano Integral Del Área Metropolitana de Mendoza (UNICIPIO).
Cuenya, Beatriz. (2012). Grandes Proyectos Urbanos, Cambios En La Centralidad Urbana y Conflictos de Intereses. Notas Sobre La Experiencia Argentina. Pp. 27-66 in Grandes proyectos urbanos: miradas críticas sobre la experiencia argentina y brasilera. Café de las ciudades.

Cuenya, Beatriz, and Manuela Corral. (2011). Empresarialismo, Economía Del 
Suelo y Grandes Proyectos Urbanos: El Modelo de Puerto Madero En Buenos Aires. EURE (Santiago) 37(111):25-45.

Cuenya, Beatriz, Eduardo González, Gustavo Mosto, and Silvia Pupareli. (2012). Movilización de Plusvalías En Un Gran Proyecto Urbano. La Experiencia de Puerto Norte, En Rosario in Grandes Proyectos Urbanos. Miradas críticas sobre la experiencia argentina y brasileña, edited by B. Cuenya, P. Novais, and C. Vainer. Buenos Aires: Café de las ciudades.

D'Inca, Maria Veronica, and Nélida Marina Berón. (2013). Expansión Urbana de Ciudades Intermedias: Modelos de Desarrollo y Legislación. Reflexión a Partir Del Caso Del 'Gran Mendoza', Argentina." Geo UERJ 1(24):256-84. doi: 10.12957/ geouerj.2013.6916.

Fiori Arantes, Pedro. (2006). O Ajuste Urbano: As Políticas Do Banco Mundial e Do BID Para as Cidades. Revista Do Programa de Pós-Graduação Em Arquitetura e Urbanismo Da FAUUSP 20:60-75.

Galván, Rodrigo Ezequiel. (2020). Análisis de La Incorporación de La Cláusula UVA En Los Créditos Del Pro. Cre. Ar. Revista Perspectivas de Políticas Públicas 10(19):43-60.

Gudiño, María Elina, Julieta Dalla Torre, and Matías Ghilardi. (2014). El Territorio Como Expresión de Las Condiciones de Vida y Las Políticas Sociales. Bitacora Urbano Territorial 24(1):1-23.

Harvey, David. (1989). From Managerialism to Entrepreneurialism: The Transformation in Urban Governance in Late Capitalism. Geografiska Annaler. Series B, Human Geography 71(1):3. doi: 10.2307/490503.

Hofman, Annelore, and Manuel B. Aalbers. (2017). "Spaces of Lobbying." Geography Compass 11(3):1-17. doi: 10.1111/ gec3.12309.

Insa, Cinthia. (2010). Desigualdad y Marginación En El Corazón de Las Ciudades y En La Periferia . El Asentamiento Costa Esperanza , Un Núcleo Urbano Segregado En El Gran Mendoza Alumna : Cinthia Insa Registro № : 16662 Dirigido Por." Universidad Nacional de Cuyo.
Jajamovich, Guillermo. (2016). La Circulación de Políticas Urbanas: La Corporación Puerto Madero y "Mendoza-Madero" Journal of Latin American Geography 15(3):67-85.

Lentini, Mercedes, Delia Palero, and Elma Montaña. (2010). La Inequidad En El Acceso Al Suelo Urbano: Los Procesos de Segregación Residencial e Informalidad Urbana. in XI Seminario Internacional RII: desigualdades territoriales. Mendoza: Red Iberoamericana de Editores de Revistas.

Magallanes, Rodrigo Martín. (2017). Urbanismo Neoliberal y Conflictos Urbanos En El Área Metropolitana de Mendoza: Transformaciones En Zonas Pericentrales de Guaymallén. Universidad Nacional de Cuyo.

Molina, Alberto. (2013). Como En Una Gran Pecera. Mendoza: EDIUNC.

Morgani, Rodolfo, and Mariana Raffani. 2012. Mendoza y Los Orígenes de La Ciudad Neoliberal. Tiempo y Espacio (28):5575. Recuperado de: http://revistas.ubiobio. cl/index.php/TYE/article/view/1794

Morgani, Rodolfo, and Pablo Rizzo. (2014). Urbanismo Neoliberal y Conflictos Urbanos En Mendoza. irenovación Urbana o Gentrificación En Los Terrenos Del Ferrocarril? Mendoza: Qellqasqa.

Navarrete, María José, Ana Laura Avila, and María Marta Bernabeu. (2017). El Regreso a La Ciudad Construida En Mendoza ¿Quiénes Regresan y Quiénes Se Van? in XXXI Congreso ALAS. Las encrucijadas abiertas de América Latina. La sociología en tiempos de cambio. Montevideo.

Pírez, Pedro, and Natalia Cosacov. (2016). El Derecho a La Ciudad y La Reestructuración Neoliberal En Buenos Aires: Movilizaciones de Sectores Populares ("Insolventes") y Clases Medias ("Solventes").

Del Río, Juan Pablo. (2017). Crédito Hipotecario, Acceso Al Suelo y Clase Media En La Implementación Del Pro.Cre.Ar. En La Ciudad de La Plata. in Detrás de los conflictos. Estudios sobre desigualdad urbana en la Región Metropolitana de Buenos Aires, edited by M. C. Cravino. Los Polvorines: Ediciones UNGS. 
Roitman, Sonia. (2000). Transformaciones Urbanas En Los $90^{\prime}$ :Los Barrios Cerrados Del Área Metropolitana de Mendoza. IV Jornadas de Sociología 1-16.

Salomone, Mariano, and Pedro Marsonet. (2011). Territorio Urbano: Espacio Para La Vida vs. Espacio Para El Capital. La Disputa Por Los Terrenos Del Ferrocarril En Mendoza. Herramienta 15(48):153-75. doi: 10.1017/CB09781107415324.004.

Sánchez, Fernanda, and Rosa Moura. (2005).Ciudades-Modelo: Estrategias Convergentes Para Su Difusión Internacional. EURE (Santiago) 31(93):21-34.

Segura, Ramiro, and Natalia Cosacov. (2019). Políticas Públicas de Vivienda: Impactos y Limitaciones Del Programa ProCreAr. Disponible en: https://revistas.unlp. edu.ar/CTyP/article/view/7371

Theodore, Nick, Jamie Peck, and Neil Brenner. (2009). Urbanismo Neoliberal: La Ciudad y El Imperio de Los Mercados. Temas Sociales SUR 66:1-12.

Vainer, Carlos. (2012). Grandes Proyectos Urbanos ¿Qué Son y Cómo Evaluarlos?" P. 388 in Grandes Proyectos Urbanos. Miradas críticas sobre la experiencia argentina y brasileña., edited by B. Cuenya, P. Novais, and C. Vainer. Buenos Aires: Café de las ciudades. Disponible en: https://www.redalyc. org/pdf/3692/369236774010.pdf

\section{Notas}

1 Licenciado en Sociología por la Universidad Nacional de Cuyo, becario doctoral del CONICET por temas estratégicos. Miembro del Instituto Multidisciplinario de Estudios Sociales Contemporáneos (IMESC), cursando el Doctorado en Ciencias Sociales de la UNCuyo.

2 Licenciado en Geografía egresado de la Universidad Nacional de Cuyo, becario CONICET. Miembro del Instituto Multidisciplinario de Estudios Sociales Contemporáneos (IMESC), cursando el Doctorado en Ciencias Sociales de la UNCuyo.
3 Mendoza es una provincia ubicada al Oeste de Argentina que cuenta con una superficie $148.827 \mathrm{~km}^{2}$ y 1.739 .000 habitantes según el último Censo Nacional de Población, Hogares y Viviendas (INDEC) en el año 2010, distribuidos principalmente en sus oasis productivos que representan el $3 \%$ del territorio provincial (oasis sur, centro y norte). La división política de la provincia se encuentra estructurada en torno a $18 \mathrm{mu}$ nicipios.

4 Para una lectura más completa sobre Puerto Madero y la corporación ver: Cuenya y Corral (2011), Nuñez, Ruiz De Gopegui (2013), Etulian (2008) y Jajamovich (2016).

5 A los fines del presente trabajo hemos agrupado en una misma dimensión las intervenciones de carácter político e institucional y a las legales e inmobiliarias (normativas inmobiliarias). En el texto original el autor las analiza como dimensiones diferentes, pero entendemos que se encuentran en estrecha relación. También cabe aclarar que no tomamos en cuenta la dimensión escalar que propone el autor.

6 CER: Coeficiente de Estabilización de Referencia: es un índice de ajuste diario, el cual es elaborado por el BCRA. Este indicador refleja la evolución de la inflación, para lo cual se toma como base de cálculo la variación registrada en el Índice de Precios al Consumidor [IPC]. EI CER se origina luego de la "pesificación" de créditos y deudas en moneda extranjera mediante el decreto $\mathrm{N}^{\circ} 214 / 2002$.

7 En mayo de 2018 se actualizaron los montos estipulados por el programa (precio de viviendas y monto del préstamo). Mientras que el subsidio por parte del Estado no se actualizó y quedó en $\$ 400.000$ por grupo familiar.

8 El desarrollo urbanístico Pro.Cre.Ar Ciudad, la "mini ciudad de la sexta sección", posee más de 1.110 departamentos en un predio de 8 hectáreas en el corazón de la ciudad a menos de diez cuadras del $\mathrm{km} 0$ de Mendoza y pretende albergar a más de 5.000 personas. Emplaza- 
do en uno de los últimos vacíos urbanos es el emprendimiento inmobiliario más importante de la provincia (público o privado) dada su posición privilegia, por la cantidad de departamentos y de metros cuadrados cubiertos y por el potencial de negocio inmobiliario que impacta en la zona.

9 Para una lectura completa sobre la historia urbana de los terrenos del ex FFCC Gral. San Martín ver: Insa, 2010; Jajamovich, 2016; Morgani \& Rizzo, 2014; Salomone \& Marsonet, 2011.

10 En mayo de 2018 el máximo de la vivienda pasó a 2,6 millones de pesos y el crédito a 1,6 millones. De esta forma las entidades bancarias pueden prestar más dinero; es decir, las familias se endeudan con más capital y a más años.

11 Esta iniciativa que surge en el 2015 propone los lineamientos y desafíos de desarrollo para el nuevo mileno, se basa en 17 Objetivos de Desarrollo Sostenible propuestos para las agendas gubernamentales y con apoyo técnico de la CEPAL.

12 Algunas agencias participantes fueron: Harvard Office for Organization, Corporación Mondragón LKS de la Universidad de Cantabria, el Instituto Superiore sui Sistemi Territoriali per L'Innovazione (Italia), entre otras.

13 Fundación Nuestra Mendoza surgió en el año 2008 promovida por tres agentes: la Asociación Civil Valos que es referente de la Responsabilidad Social Empresaria de Mendoza, la Fundación Avina que trabaja a escala latinoamericana para producir cambios para el desarrollo sostenible en la región y el diario Los Andes que es el principal medio de prensa escrita de Mendoza encargado de divulgar los resultados.

14 Los datos referidos al proyecto fueron obtenidos del Master Plan ganador (Horizonte GS), documentos oficiales de la Municipalidad de Capital y del Colegio de Arquitectos de Mendoza. 